\title{
Accelerating the Transient Simulation of Semiconductor Devices Using Filter-Bank Transforms
}

\author{
Masoud Movahhedi and Abdolali Abdipour \\ Microwave/mm-wave \& Wireless Communication Research Lab. \\ Department of Electrical Engineering, AmirKabir University of Technology, Tehran, Iran. \\ Phone: +98-21-646 6009, E-mail: movahhedi@aut.ac.ir, abdipour@aut.ac.ir
}

\begin{abstract}
Using fully or semi-implicit schemes to solve hydrodynamic transport equations is very suitable and efficient for transient simulations of semiconductor devices. But, using these techniques leads to a large system of linear equations. Here for the first time, a preconditioning method based on the filter-bank and wavelet transforms is used to facilitate the iterative solution of this system. As the first step in the performance investigation of this preconditioner in the simulation of semiconductor devices, we apply it to the modified Poisson's equation in Drift-Diffusion model. Numerical results show that the condition number are significantly reduced and convergence rate is increased. The most important advantage of this preconditioner rather than the other preconditioners is its low computational complexity which can be reduced to $O(N)$.
\end{abstract}

\section{INTRODUCTION}

In microwave and high-frequency semiconductor devices, submicrometer dimensions are used. As device sizes continue to decrease, nonlocal, hot carrier transport becomes dominant and can no longer be ignored. Therefore, hydrodynamic transport equations which are obtained by taking the first three moments of the Boltzmann Transport Equation (BTE) in conjunction with the Poisson's equation, must be used to study nonstationary transport effects in submicrometer devices [1]. These equations form a set of nonlinear, coupled and timedependent partial differential equations. The solution methods can be divided into two categories: coupled (Newton) or decoupled (Gummel) solvers [2]. The major advantage of using a coupled scheme is that there is no limit on the maximum allowable time step, $\Delta t$. However, for large $\Delta t$, the initial guess of the solution in general turns out to be very different from the true solution, and hence the overall convergence rate slows down. In addition, since the equations are solved simultaneously, CPU memory requirement is higher three times than that for a decoupled solver. The decoupled method (Gummel algorithm), treats each of the differential equations separately by decoupling the equations and solving the system step by step. Then this sequence is iteratively repeated until self-consistent values for all unknown variables are obtained with the desired accuracy. In conventional decoupled methods, the fully implicit, semi-implicit and explicit methods are used to solve the equations. Although in explicit scheme, variables can be evaluated easily through simple algebraic computations, but the major disadvantage of this scheme is that this method is numerically unstable for $\Delta t$ greater than the maximum

${ }^{1}$ This work was supported in part by Iran Telecommunication Research Center (ITRC). allowed values. For most practical problems, the maximum $\Delta t$ is very small and it makes the explicit methods to be very inefficient, especially for obtaining steady-state solutions. In fully or semi-implicit schemes, the method is unconditionally stable for any $\Delta t$ or for very large $\Delta t$ compared to explicit schemes. The only disadvantage of this scheme is its high computational cost. Even though this technique is computationally more expensive compared to explicit methods for obtaining variables in new time, it becomes more and more economical in problems where steady-state solutions or transient solutions for long times are desired, because of the possibility of using large $\Delta t$ when employing the implicit schemes.

As it has been mentioned in literature [2], using the fully and semi-implicit methods for parabolic or hyperbolic equations lead to a system of linear equations, $A x=b$. In many problems of semiconductor device simulation, size of the matrix $A$ is very large and has a large condition number. Solutions of such large systems, by a direct method is prohibitively expensive; because, these methods work fine only for well-posed problems (problems in which the corresponding matrix $A$ has a small condition number). In this case, an iterative technique is usually adopted and effective preconditioner of the matrix $A$ is required in order to make it better conditioned than the original matrix [3]. Generally, the better-conditioned system leads to an accelerated convergence in the iterative solution [4]. Some well-documented preconditioning methods such as incomplete LU factorization (ILU) and polynomial preconditioning methods [5]-[6] can be effective. However, they usually require well-above $O(N)$ operations to implement. Recently, an interesting preconditioning method based on the filter-bank and wavelet transforms was proposed [7]. The most important advantage of the new preconditioner is its low computational cost, which can be reduced to $O(N)$ complexity. In this paper we will use this preconditioner to accelerate the transient simulation of semiconductor devices. This method seems to provide efficient preconditioners for matrices arising from finite difference of PDEs. Hence, this preconditioner can be applied to matrices arising from discretizing when using the fully and semi-implicit finite difference schemes for solving time-dependent hydrodynamic transport equations. Here, as the first step in the performance investigation of this preconditioner in the simulation of semiconductor devices, we apply it to the descritized modified Poisson's equation in DriftDiffusion model. In [8], the proposed method has been applied to the standard Poisson's equation as an elliptic equation. 


\section{IMPLICIT SCHEME FOR SEMICONDUCTOR EQUATIONS}

Consider a time-dependent equation of the following form:

$$
\frac{\partial x}{\partial t}=f(x) .
$$

If a Taylor expansion of $x$ is made around $x$ at $t=t_{1}+1$, then we have

$$
x^{t_{1}+1}=x^{t_{1}}+\Delta t \cdot\left(\frac{\partial x}{\partial t}\right)_{t_{1}+1}+O\left(\Delta t^{2}\right) .
$$

Using (1) and (2) we obtain

$$
\frac{x^{t_{1}+1}-x^{t_{1}}}{\Delta t} \cong f\left(x^{t_{1}+1}\right) .
$$

Application of the difference approximation to operator " $f$ ", gives

$$
(I-\Delta t A) x^{t_{1}+1}=x^{t_{1}}
$$

where $A$ is the difference approximation of operator " $f$ ". It can be shown that the above scheme, known as implicit method, when applied to the semiconductor equations, is stable for very large $\Delta t$ compared to the explicit method [2].

Here, without loss of generality, we can consider DriftDiffusion model and corresponding equations which are as the following for an unipolar device [12]:

$$
\begin{gathered}
\frac{\partial n}{\partial t}+\nabla \cdot(n \vec{v})=0 \\
\nabla^{2} \psi=-\frac{q}{\epsilon_{0} \epsilon_{r}}\left(N_{D}^{+}-n\right),
\end{gathered}
$$

where $n \vec{v}=-n \mu \vec{E}-\vec{\nabla}(D n)$ and when defining carrier mobility as $\mu \equiv q \tau_{p} / m^{\star}$ and Diffusion coefficient as $D \equiv$ $\mu k T / q$.

\section{A. Modified Poisson's Equation}

While solving for the potential $\psi^{t+1}$ at time level $(t+1)$, the carrier concentration $n^{t+1}$ at $(t+1)$ ia not available. But this can be approximated in the following ways [2]:

$$
\nabla^{2} \psi^{t+1}=-\frac{q}{\epsilon_{0} \epsilon_{r}}\left(N_{D}^{+}-n^{t+1}\right) .
$$

Expanding $n^{t+1}$ using a Taylor series gives

$$
n^{t+1}=n^{t}+\Delta t \cdot\left(\frac{\partial n}{\partial t}\right)_{t}+O\left(\Delta t^{2}\right) .
$$

Ignoring second and higher order terms in time, $O\left(\Delta t^{2}\right)$, and substituting for $(\partial n / \partial t)_{t}$ from the continuity equation yields

$$
n^{t+1}=n^{t}+\Delta t \nabla \cdot\left(-n^{t} \mu \vec{\nabla} \psi^{t+1}+\vec{\nabla}\left(D n^{t}\right)\right) .
$$

Hence, (6) can be rewritten as follows:

$$
\begin{gathered}
\nabla^{2} \psi^{t+1}\left(1+\frac{q \mu \Delta t}{\epsilon} n^{t}\right)+\frac{q \mu \Delta t}{\epsilon} \vec{\nabla} \psi^{t+1} \cdot \vec{\nabla} n^{t}= \\
\frac{q}{\epsilon}\left(-N_{D}+n^{t}+D \Delta t \nabla^{2} n^{t}\right) .
\end{gathered}
$$

The modified Poisson's equation, (10), is solved for $\psi^{t+1}$ using the full implicit method.

\section{B. Carrier Continuity Equation}

For solving carrier continuity equation by an implicit scheme, it is considered as:

$$
\frac{\partial n}{\partial t}=\nabla \cdot\left(\mu n^{t+1} \vec{E}^{t+1}+D \vec{\nabla} n^{t+1}\right)
$$

or

$$
\frac{n^{t+1}-n^{t}}{\Delta t}=\mu \vec{E}^{t+1} \cdot \vec{\nabla} n^{t+1}-n^{t+1} \mu \nabla^{2} \psi^{t+1}+D \nabla^{2} n^{t+1} .
$$

In above equation, it is assumed that $\psi^{t+1}$ is known. The above assumption is justified, since $\psi^{t+1}$ is available once (10) is solved. Equation (11) is used to solve $n^{t+1}$.

\section{Filter-BAnK BASEd Preconditioner}

Most of the orthogonal wavelet-based preconditioners provide effective schemes for matrices based on the structure of the matrices themselves rather than relying on detailed knowledge of the underlying problem from which they arise [9]. Here, we consider that the matrix, $A$, comes from the discretization of a PDE. This leads to better approximation of the transformed matrix with lower computational cost for preconditioning which can be reduced to $O(N)$ complexity [7]. Results show that general operators have a sparse representation in wavelet bases were derived in [10]. In [11] it was shown that the efficient decomposition level of the wavelet transform can be used to construct diagonal preconditioners when using a Galerkin method. The discrete analogue of the biorthogonal wavelet transforms relies on so called perfect reconstruction filter-bank transforms. In this paper we use biorthogonal filter-bank transforms and follow the preconditioner algorithm for discretized PDE's explained in [7]-[8]. Here we apply this algorithm to carry on the construction of preconditioner for the modified Poisson's equation, which is shown to improve the performance of the method. The motive for using orthogonal wavelet transforms is that the condition numbers of the transformed and untransformed operators are the same. However, the condition number actually decreases when doing biorthogonal filter-bank transforms.

\section{A. Construction of the Preconditioner}

If we consider a partial differential equation and assume that this problem is discretized with a finite difference method, a system of linear equations can be obtained as $A x=b$. We want to use the filter-bank transform to precondition the operator, $A$. If the filter-bank transform of matrix $A$ is called $\tilde{A}$, its matrix representation is as shown in Fig. 1. Here, $M$ is the approximation of the transformed operator. From its inverse which can be computed very easily, we will construct the preconditioner [8].

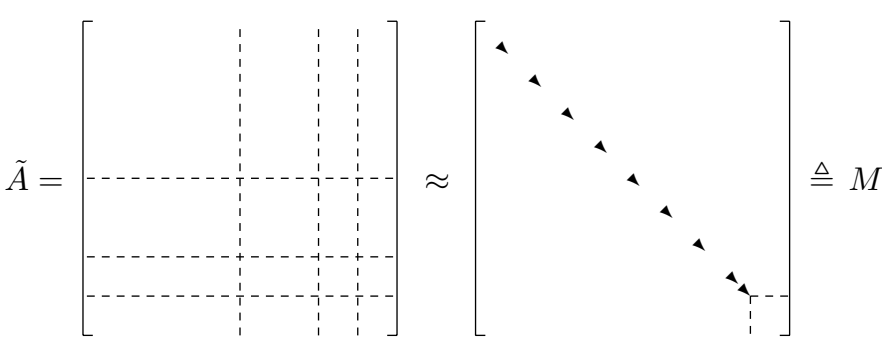

Fig. 1. Structure of matrix representation of transformed operator. 


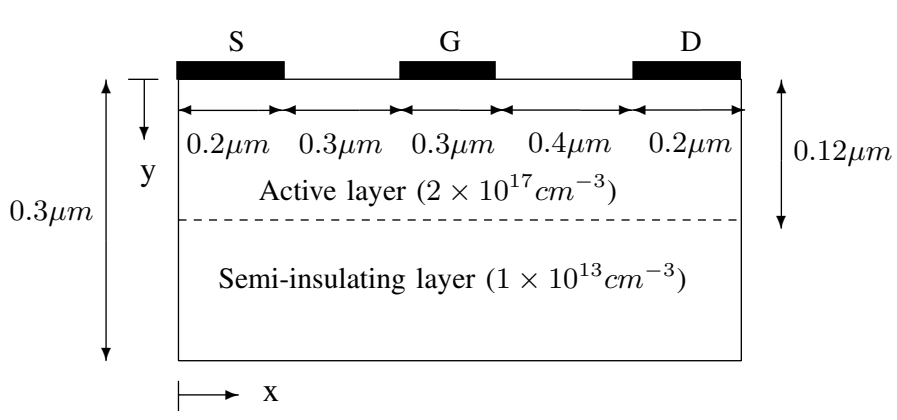

Fig. 2. Cross section of the simulated MESFET transistor.

\begin{tabular}{|c|c|c|c|}
\hline Type of filter bank & Name & {$[\mathbf{M}]$} & Condition number \\
\hline \hline$\delta_{1}$ & Delta $1_{22}$ & $\left(2^{4} .2^{3}\right) \times\left(2^{4} .2^{3}\right)$ & 2099 \\
\hline$\delta_{1}$ & Delta $1_{32}$ & $\left(2^{3} .2^{3}\right) \times\left(2^{3} .2^{3}\right)$ & 2528 \\
\hline$\delta_{1}$ & Delta $1_{33}$ & $\left(2^{3} .2^{2}\right) \times\left(2^{3} .2^{2}\right)$ & 2694 \\
\hline$\delta_{1}$ & Delta $1_{43}$ & $\left(2^{2} .2^{2}\right) \times\left(2^{2} .2^{2}\right)$ & 3381 \\
\hline$\delta_{3}$ & Delta $3_{22}$ & $\left(2^{4} .2^{3}\right) \times\left(2^{4} .2^{3}\right)$ & 2738 \\
\hline$\delta_{3}$ & Delta $3_{32}$ & $\left(2^{3} .2^{3}\right) \times\left(2^{3} .2^{3}\right)$ & 3448 \\
\hline$\delta_{3}$ & Delta $3_{33}$ & $\left(2^{3} .2^{2}\right) \times\left(2^{3} .2^{2}\right)$ & 3759 \\
\hline$\delta_{3}$ & Delta $3_{43}$ & $\left(2^{2} .2^{2}\right) \times\left(2^{2} .2^{2}\right)$ & 4786 \\
\hline
\end{tabular}

TABLE I

Condition Number of the Preconditioned Matrix CORRESPONDING TO THE MESFET STRUCTURE FOR DIFFERENT FiLTER-BANK TRANSForms.

\begin{tabular}{|c|c|c|c|}
\hline Type of wavelet & Name & {$[\hat{\mathbf{M}}]$} & Condition number \\
\hline$\overline{\overline{D_{4}}}$ & $\overline{D a b 4_{22}}$ & $\left(2^{4} .2^{3}\right) \times\left(2^{4} .2^{3}\right)$ & 3976 \\
\hline$D_{4}$ & $D a b 4_{32}$ & $\left(2^{3} .2^{3}\right) \times\left(2^{3} .2^{3}\right)$ & 3248 \\
\hline$D_{4}$ & $D a b 4_{33}$ & $\left(2^{3} .2^{2}\right) \times\left(2^{3} .2^{2}\right)$ & 3246 \\
\hline$D_{4}$ & $D a b 4_{43}$ & $\left(2^{2} .2^{2}\right) \times\left(2^{2} .2^{2}\right)$ & 3199 \\
\hline$D_{2}($ Haar $)$ & $D a b 2_{22}$ & $\left(2^{4} .2^{3}\right) \times\left(2^{4} .2^{3}\right)$ & 6664 \\
\hline$D_{2}($ Haar $)$ & $D a b 2_{32}$ & $\left(2^{3} .2^{3}\right) \times\left(2^{3} .2^{3}\right)$ & 5783 \\
\hline$D_{2}($ Harr $)$ & $D a b 2_{33}$ & $\left(2^{3} .2^{2}\right) \times\left(2^{3} .2^{2}\right)$ & 5634 \\
\hline$D_{2}($ Harr $)$ & $D a b 243$ & $\left(2^{2} \cdot 2^{2}\right) \times\left(2^{2} .2^{2}\right)$ & 5454 \\
\hline
\end{tabular}

TABLE II

Condition Number of the Preconditioned Matrix CORRESPONDING TO THE MESFET STRUCTURE FOR DIFFERENT WAVELET TRANSFORMS. distribution, $n^{t}$. Also, this situation is established in implicit discretization of carrier continuity equation. It is important to note, when an explicit scheme is used to solve Drift-Diffusion model equations, the maximum allowable time step is about $\Delta t_{\text {explicit }}=0.001 \mathrm{ps}$. But when the equations are discretized by the above implicit scheme, the method will be stable for very large $\Delta t$; for instance $\Delta t=100 \Delta t_{\text {explicit }}$.

In this study, the proposed method is applied to matrix $A$ when $t=0$, i.e. $n=N_{D}$, and $\Delta t=0.01 \mathrm{ps}$. The condition number of this matrix is 11607 and we apply the proposed filter-bank based preconditioner to it to reduce its condition number. In Tables I and II, we present the variation of condition number of the preconditioned matrix according to the type of filter-bank and wavelet transforms and to the number of steps in the transform, which determines the size of nondiagonal part of matrix $M$ called $M$. We have used the tensor product (2-D) transforms for preconditioning. It is clearly shown that for the $\delta$ filter-bank transforms, the condition number decreases as the filter-bank is of lower order and as the decomposition levels decrease (Table I). But for the Daubechies wavelet transforms the condition number decrease as the wavelet is as higher order and as the decomposition levels increases (Table II).

Fig. 3 shows the convergence behavior of the proposed preconditioner for different filter-bank and wavelet 2-D transforms. Convergence behavior of the preconditioned system is similar to the variation of its condition number. As it is seen, the convergence rate increases as the filter-bank is of lower order and as the decomposition levels decreases. But for the Daubechies wavelet transforms, the convergence rate increases as the wavelet is as higher order and as the decomposition level increases. We found that preconditioning using $\delta_{1}$ filter-bank transform converges faster than the other filter-bank and wavelet transforms. By increasing the number of steps in the transform, the size of nondiagonal part of
$M$ decreases. Therefore, the computational complexity of the preconditioning method, which is equal to $O\left(N+M^{3}\right)$ can be reduced by increasing the number of steps in the transform [8]. It is interesting that we can obtain both good conditioning and low computational cost by using $\delta_{1}$ filterbank transform. For example, in case Delta $1_{43}$, the complexity equals to $O(N)$ [8]. To compare the performance of the used preconditioner (filter-bank based preconditioner) with the well-known preconditioning methods, the convergence rate of the incomplete LU factorization (ILU) preconditioner which applied to our problem, has been illustrated in Fig. 3. Although the performance of the used preconditioner is better when applied to the Laplacian operator matrix in standard poisson's equation [8], for $\delta_{1}$ filter-bank transform with different decomposition levels, the convergence rate is faster than the $\operatorname{ILU}(0)$ preconditioner.

Here, we use the solution of the modified Poisson's equation obtained using the proposed filter bank preconditioner for simulation of the considered MESFET transistor. Fig. 4 shows the potential distribution obtained using the proposed algorithm, while Fig. 5 illustrates the carrier density distribution. It is significant to indicate that the proposed algorithm gives precisely the same results obtained when the used iterative method does not employ the proposed preconditioner method.

\section{CONCLUSiON}

In this paper, we have proposed to use a filter-bank based preconditioner for accelerating the iterative solutions of the large systems. These systems of linear equations arise from an implicit discretization of semiconductor equations. The performance of the preconditioner when applied to the modified Poisson's equation has been investigated. Results show that 


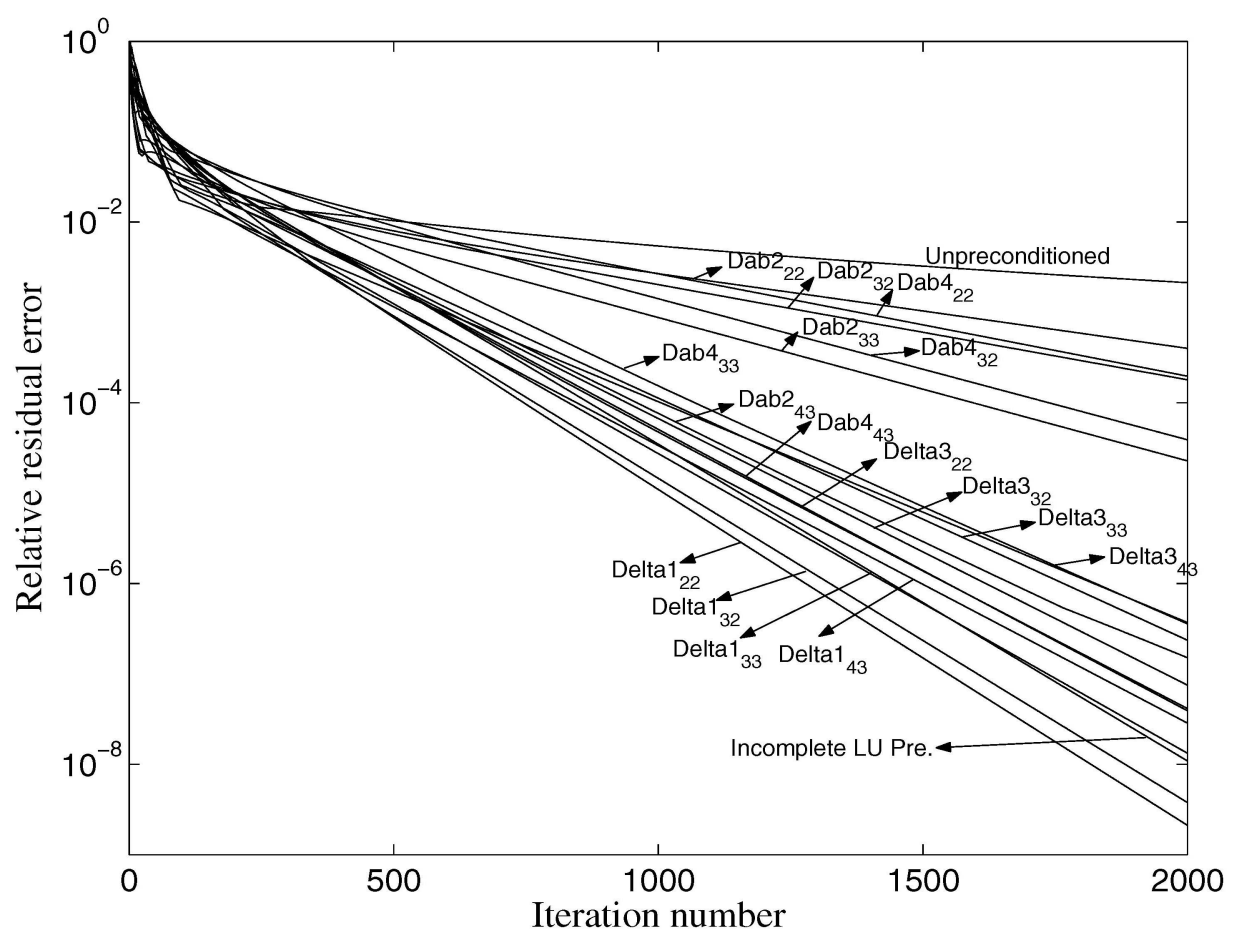

Fig. 3. Convergence behavior of the ILU preconditioner and the proposed preconditioner system by different wavelet and filter-bank transforms.

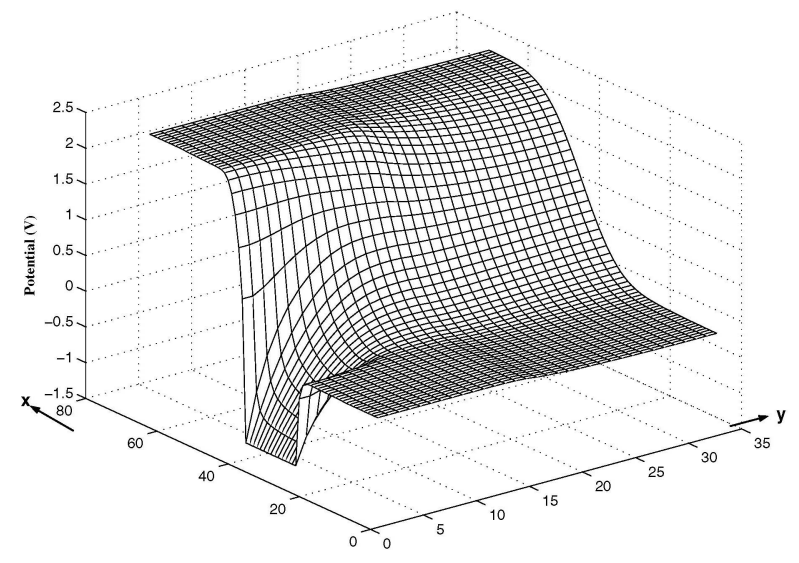

Fig. 4. Steady-state potential distribution obtained using the proposed algorithm.

the convergence rate of the used preconditioning scheme, by $\delta_{1}$ filter-bank transform, is faster than the well-known ILU method. Moreover, the computational cost of the considered method is as low as $O(N)$ which is very better than the other methods.

\section{REFERENCES}

[1] T. Grasser, T. W. Tang, H. Kosina, and S. A. Selberherr, "A review of hydrodynamic and energy-transport models for semiconductor device simulation," Proceedings of the IEEE, vol. 51, no. 8, pp. 251-274, Aug. 2003.

[2] S. Yoganathan, and S. K. Banerjee, "A new decoupled algorithm for nonstationary, transient simulations of GaAs MESFET's" IEEE Trans. Electron Devices, vol. 39, no. 7, pp. 1578-1587, Jul. 1992.

[3] Y. Saad, Iterative methods for sparse linear systems. PWS Publishing Co., Boston, 1996.

[4] H. Deng, and H. Ling, "An Efficient Wavelet Preconditioner for Iterative Solution of Three-Dimensional Electromagnetic Integral Equations," IEEE Trans. Antennas and Propagation, vol. 51, pp. 654-660, March 2003.

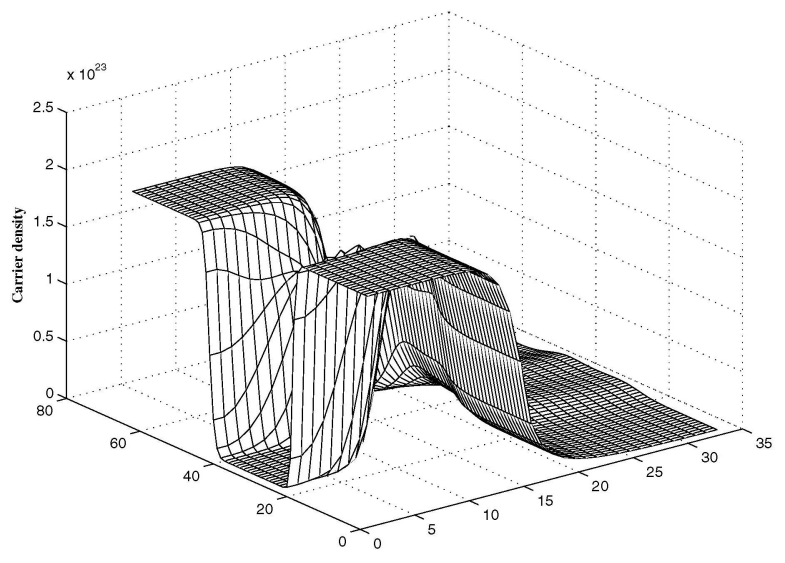

Fig. 5. Steady-state carrier density distribution obtained using the proposed algorithm.

[5] A. M. Bruaset, A Survey of Preconditioned Iterative Methods. New York: Wiley, 1995.

[6] R. Weiss, Parameter-Free Iterative Linear Solvers. Berlin, Germany: Akademie Verlag GmbH, 1996.

[7] J. Walen, "Filter bank preconditioners for finite difference discretizations of PDEs," Technical Report 198, Dep. of Scientific Computing, Uppsala University, Sweden, July 1997.

[8] M. Movahhedi, and A. Abdipour, "Improvement of Active Microwave Device Modeling Using Filter-Bank Transforms," in Proceedings of the 35th European Microwave Conference (EuMC2005), Paris, France, October 2005.

[9] J. M. Ford, Wavelet-based preconditioning of dense linear systems, $\mathrm{PhD}$ thesis, University of Liverpool, 2001.

[10] G. Beylkin, R. Coiman, and V. Rokhlin, "Fast wavelet transform and numerical algorithms I.," Comm. Pure Appl. Math., vol. 44, pp. 141-148, 1991.

[11] S. Jaffard, "Wavelet method for fast resolution of elliptic problems," SIAM J. Numer. Anal., vol. 29, pp. 965-986, 1992.

[12] Y. K. Feng, and A. Hintz, "Simulation of sub-micrometer GaAs MESFET's using a full dynamic transport model," IEEE Trans. Electron Devices, vol. 35, pp. 1419-1431, September 1988. 